\title{
Synchrophasor Based Monitoring System for Grid Interactive Energy Storage System Control
}

\author{
Roozbeh Torkzadeh ${ }^{1(\bowtie)}\left(\mathbb{D}\right.$, Mojtaba Eliassi $^{1}$, Peyman Mazidi $^{1}$, \\ Pedro Rodriguez ${ }^{1,2}$, Dalibor Brnobić ${ }^{3}$, Konstantinos F. Krommydas ${ }^{4}$, \\ Akylas C. Stratigakos ${ }^{4}$, Christos Dikeakos ${ }^{5}$, Michalis Michael ${ }^{6}$, \\ Rogiros Tapakis ${ }^{6}$, Vasiliki Vita ${ }^{7}$, Elias Zafiropoulos ${ }^{7}$, \\ Ricardo Pastor ${ }^{8}$, and George Boultadakis 9 \\ ${ }^{1}$ Research Institute of Science and Technology, \\ Loyola University Andalusia, Seville, Spain \\ rtorkzadeh@ieee.org \\ 2 Research Center on Renewable Electrical Energy Systems, \\ Technical University of Catalonia, Barcelona, Spain \\ ${ }^{3}$ Studio Elektronike Rijeka (STER d. o. o), Rijeka, Croatia \\ ${ }^{4}$ Department of Electrical and Computer Engineering, \\ University of Patras, Rion, Greece \\ 5 Independent Power Transmission Operator (IPTO TSO), Athens, Greece \\ ${ }^{6}$ Transmission System Operator, Cyprus (TSOC), Strovolos, Cyprus \\ 7 Institute of Communications and Computer Systems, \\ 9 Iroon Polytechniou Street, 15780 Athens, Greece \\ ${ }^{8}$ Centro de Investigação em Energia REN - State Grid, S.A, Sacavém, Portugal \\ ${ }^{9}$ European Dynamics Luxembourg SA., 12, Rue Jean Engling, 1466 \\ Luxembourg, Luxembourg
}

\begin{abstract}
Energy Storage Systems installed at primary substations can be used by different participants of the power system for handling the emerging uncertainties caused by supply-side variations, demand-side flexibility and grid topology changes. This work presents a practical design for the monitoring system of the controller of the grid interactive energy storage system. This monitoring scheme takes advantage of synchrophasor measurements gathered by phasor measurement units of wide area measurement systems. The analysis of synchrophasor measurements provides real-time situational awareness over the status of the grid. Therefore, the integration of synchrophasor measurements into the control loop of GI-ESS will enable them to participate in power services of the flexibility market. Furthermore, the implementation of a basic power oscillation damping function as an example of power services using the proposed monitoring system is illustrated in this paper.
\end{abstract}

Keywords: Phasor measurement unit · Grid interactive battery energy storage system $\cdot$ Wide area control system 


\section{Introduction}

Power systems all over the world are facing significant changes by different drivers that are changing the conditions of system operation. The significant increase of renewable resources is one of these drivers [1]. Due to their intimate nature, they induce more uncertainty to the grid, which needs more severe operation. The other game changer is the evolving of modern distribution networks. These systems become more complicated, regarding their new role in generation. Today, these grids can be known as key infrastructures both in consumption and generation because of the large presence of low and medium voltage connected distributed energy resources (DERs) and prosumers such as electric vehicles and energy storage systems (ESSs) [2].

On the one hand, in order to cope with these changes, distribution and transmission system operators (DSOs and TSOs) should incorporate different controllable equipment (CE) into the grid [3]. Generation units, FACTS devices, phase shifting transformers and ESSs are examples of these controllable resources. On the other hand, for guaranteeing the flexible operation of the grid, maintaining the system reliability and exploiting the efficient use of sustainable energy, the development of advanced monitoring solutions and control frameworks is necessary both for distribution and transmission grids [4].

In [5], an active/reactive power control strategy for ESS is proposed that takes into account the required profile of power for electrical grid by using local measurements. The authors of [6] propose a hysteresis-based PI-state control scheme for solving the problem of the limited fault ride-through capability of grid-connected DFIGs during faults and disturbances. They use local measurements for controlling the converter, which is connected to the ESS. The authors of [7] propose a novel parallel control for modular ESSs through the comparative analysis of various conventional parallel types of control by using local measurements for their proposed control scheme. Furthermore, in [8], the authors consider a distributed ESS-based control paradigm for enhancement of the power systems transient stability. They use local measurements (sensors) in a multi-agent control framework that includes both synchronous generators and ESSs. Kiaei and Lotfifard propose a model predictive control (MPC) method to improve the power systems transient stability by controlling the state of charge and discharge of ESS installed throughout power systems by using local measurements [9].

In [10], the authors use ESS for the transmission grid congestion management. In addition, they assess the effects of transmission congestion on the profitability of arbitrage by ESS in the electricity markets. Authors of [6] propose a scalable wide area coordinated control scheme by using distributed MPC on ESS to improve the rotorangle stability in cases of large disturbances of the power system. Furthermore, they implement a local measurement system for the controllers of ESS in each area; however; the controllers are just able to perform coordinated control schemes. This coordinated control scheme is based on data exchange links.

The presented monitoring structures for control of ESS in previous works have just focused on a single control scheme; however; it is essential for grid scale ESSs to participate in different power services to make them financially feasible [11]. Therefore, this work takes advantage of wide area measurement system (WAMS) to present a 
configurable monitoring schemes for control of grid interactive ESS (GI-ESS). This will empower GI-ESS to participate in different power services in flexibility market, as well as, cover the drawbacks of decentralized, distributed and hierarchical control architectures.

In this paper, GI-ESS is referred to a grid scale ESS that is installed at primary substation. Therefore, it could be a representative for a single node in the well-known concept of active distribution networks [12]. Regarding the position of active substations as the interface with the transmission and distribution grids, they can participate in real-time operation enhancement of both girds. Overall, the main contributions of this paper are described as follows:

1. This works presents a monitoring scheme for the control of GI-BESS based on the synchrophasor measurements gathered by WAMS.

2. The provided situational awareness over the grid will enable the GI-BESS to participate in different power services in the flexibility market.

3. A basic power damping oscillation (POD) function is presented as an instance for power services that can be delivered by the GI-ESS.

The rest of this paper is organized as follows. Section 2 presents the main ideas behind the work and the proposed WAMS-based monitoring system for GI-BESS. The detailed design of the proposed monitoring system for POD function is illustrated in Sect. 3. The simulation results are presented in Sect. 4, and finally, Sect. 5 concludes the paper.

\section{Synchrophasor Measurements Integration into Control}

Monitoring systems are essential parts of every closed-loop control system. As it discussed in [11], many works can be found that take advantage of synchrophasor measurements into their control schemes; however; limited works can be found which have focused on the architecture of the monitoring system.

\subsection{Synchrophasor Measurement Technologies}

Synchrophasor Measurement Technologies (SMT) and their applications are encouraging solutions for the monitoring of emerging power system [13]. SMT can provide a wide range of real-time, online and offline applications such as stability assessment, congestion management and model validation for all monitoring, protection and control of the grid [14-16]. Generally, SMT includes phasor measurement units (PMUs), WAMS, and their applications in power systems monitoring and control.

PMU is an advanced technology in terms of measurements in power systems. This device is capable of gathering real-time measurement of voltage and current phasor (magnitude and angle), as well as, frequency and rate of change of frequency in a time synchronized manner. Precise time synchronized measurements characterized by GPS time tags and hybrid frequency and phase estimation algorithms are the major aspects that represent a substantial improvement in the concept of AC quantity measurement and provide power system operators many useful applications. Today, operation and 
planning of large power systems are enabled by the use of operation infrastructure, advanced measurement technology and information tools that WAMS provides. Typically, power system operators use WAMS as a standalone system. Therefore, these days, WAMS defined as a complimentary monitoring system beside SCADA, which is the conventional tool for power system operation and control.

For this purpose, the main function of WAMS is enhancing the real-time "situational awareness" of grid operator's that is essential for safe and reliable operation of the system. Furthermore, WAMS also supports post-event analysis for significant disturbances that occurs in the system. It is expected that WAMS and SMT become integrated into grids' real-time control centres (CC) in the near future [17].

Unlike conventional monitoring systems such as SCADA, energy management system (EMS) and substation monitoring system, SMT can be used in wide variety of control schemes including communication-based (centralized, decentralized and distributed) and autonomous (or local) control schemes, as well as, any hybrid combination of these schemes. Figure 1 shows different monitoring systems regarding their applications in control schemes and phenomena monitoring window. Regarding this figure, it could be note that:

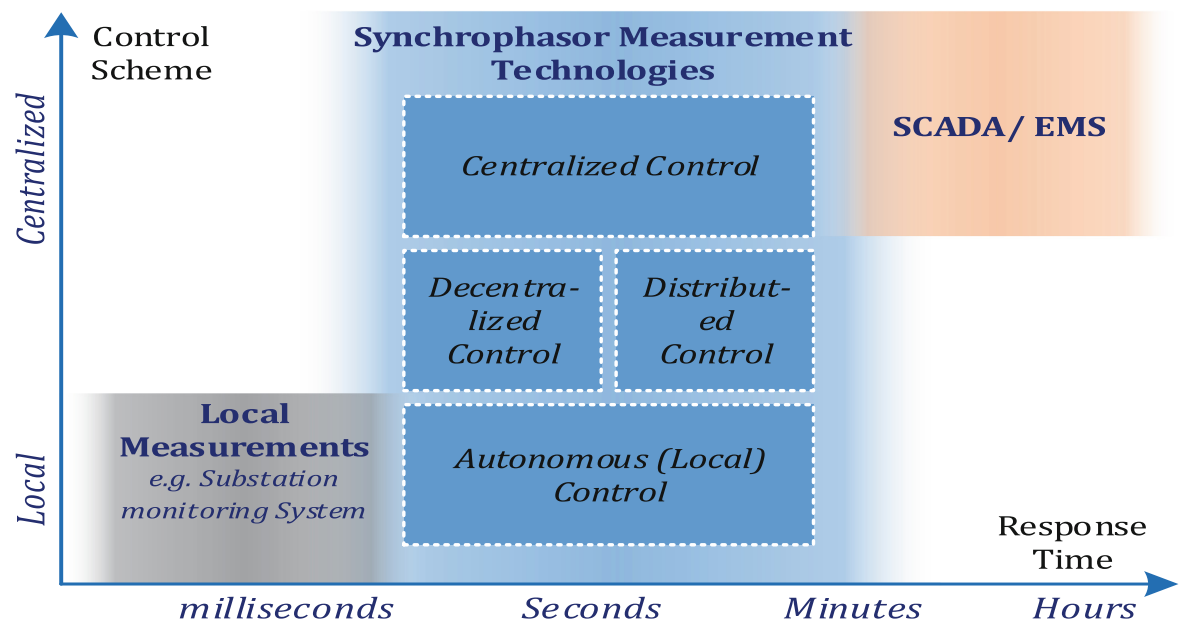

Fig. 1. Monitoring systems regarding time response and available control schemes.

- SMT can detect phenomena within the range of milliseconds to some minutes; however; there are modern PMUs that supports slow mode recording to detect phenomena with much lower occurrence frequency.

- Local measurements, such as substation monitoring systems are designed to provide measurements for local control loops. In addition, they can provide measurements for protective relays in substations and log the switching events, as well as, faults waveforms in the substation logging data centre.

- A typical RTU can gather and send electrical quantities such as voltage magnitude, active and reactive power in range of 1 to $5 \mathrm{~s}$. The SCADA system receives and 
archives these values. In addition, visualization part of SCADA system provides information about the status of the grid and its equipment for the operators in the CC.

- Generally, EMS uses the data gathered by SCADA system and smart meters for managing the energy production and consumption in the grid.

\subsection{General Structure of SMT Integration into GI-ESS Control}

In this paper, PMU and WAMS are taken into account as the local and wide area sources of synchrophasor measurements in concept of SMT.

The results of measurements-based algorithms such as oscillation detection, online transient stability assessment, online voltage stability assessment which can be implemented in local PDCs within power substations or CCs [18] are the sources of developed knowledge for SMT. This knowledge is extracted based on the synchrophasor measurements collected by WAMS or any other hybrid source of data. This integration provides situational awareness over the grid for the GI-ESS controller to enable it participation in dynamic stability improvement.

General structure is presented for SMT integration into control of GI-ESS in Fig. 2. This general structure shows how the local GI-ESS Controller can control the ESS Converter within the active substation, and how it can receive data from different monitoring systems to set the reference values for injecting/absorbing active and reactive power of the ESS.

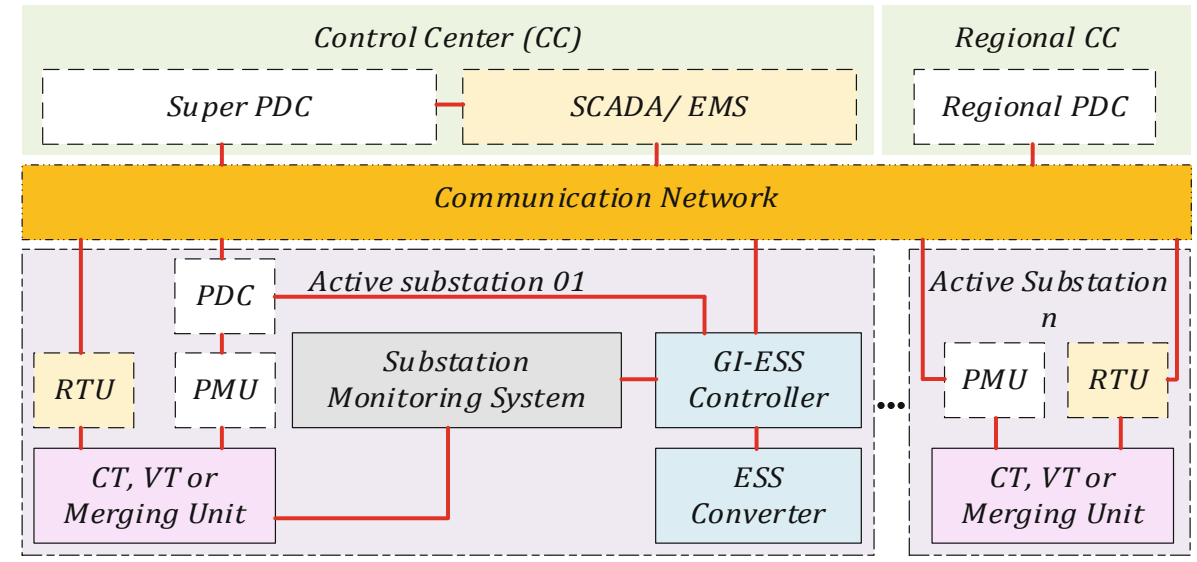

Fig. 2. General structure of SMT integration into GI-ESS control.

Regarding this general structure, different autonomous and communication-based control schemes can be implemented for the GI-ESS controller [12]. In this general structure, it is possible for GI-ESS to participate in inter-area interaction such as interarea oscillation damping because of the available communication link between different Regional CCs. 


\subsection{Proposed WAMS-Based Solution for Monitoring System}

Figure 3 shows the structure of WAMS-based solution for monitoring system. Regardless of the implemented WAMS architecture (hierarchical, centralized or hybrid), in the presented design, WAMS provides a historical database and a real-time stream of synchrophasor measurements of whole system.

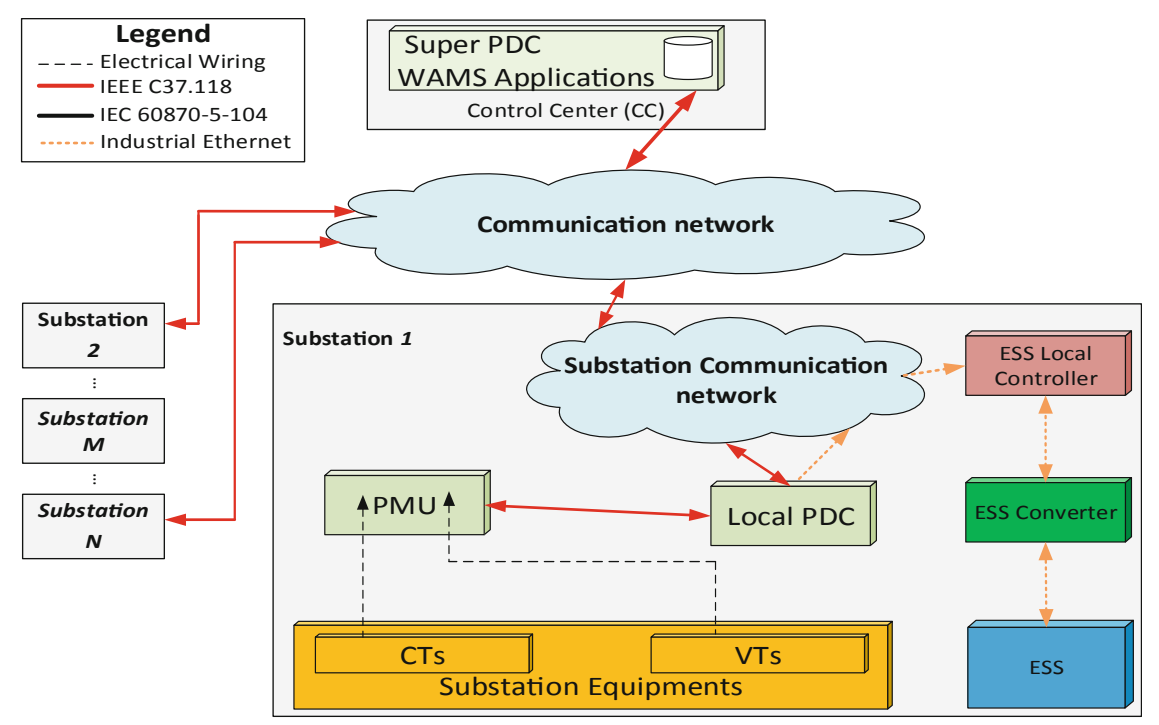

Fig. 3. WAMS-based solution for control.

In Fig. 3, CTs and VTs provide suitable signals for PMU, then, the PMU sends the synchrophasor measurements to the local PDC (if applicable) or Super PDC. Wide area applications in CC or local synchrophasor measurement-based applications such as oscillation detection and online transient stability assessment on local PDC provide the knowledge over the grid status. This extracted knowledge over the grid is transmitted to ESS local controller through the communication link.

The communication protocol between PMU, Local PDC (if applicable) and super PDC is IEEE C37.118. It should be noted that, regarding the results of PMU placement studies, PMUs are located on specific substations to make the voltage and current of specific lines observable, consequently, there could be some substations in the area (e.g. Substation M) without any installed PMUs.

ESS local controller can send the reference values for exchanging active and reactive power to the ESS converter regarding the grid and ESS status. For instance, the received knowledge over the grids identifies a local oscillation mode in the area of the GI-BESS, which enables the controller of GI-BESS to calculate the exact amount of 
active power, which is needed to be injected for damping of that specific mode. If the storage can provide that amount of active power, regarding the status of stored energy, the GI-BESS can participate in flexibility market for this power service.

One of the outmost aspect of this design is the need for implementation of real-time Ethernet protocol. The reason for this protocol selection is the need of deterministic communication link implementation in the closed-loop control systems. Because of this reason, it is essential to implement the real-time deterministic communication channel between each active substation and CC.

In addition, strict time stamping of PMU data can be used in control algorithms to suppress variations in communication channels for synchronization of local control loop to (global) WAMS measurement.

\section{WAMS-Based Power Oscillation Damping}

Low frequency inter-area oscillations can limit the power transfer between areas of power system [19]. Therefore, it is essential to increase the maximum power transfer $\left(P_{\text {Max }}\right)$ between areas. Below, the structure of proposed controller for POD function is discussed.

Based on [20, 21], the objective of the Control Lyapunov Function (CLF) is to choose the value of a control variable in such a manner to make the output of the CLF non-positive. This is to ensure the stability of the system.

In this study, the WAMS delivers system-wide frequency and voltage angle measurements to the POD controller of the GI-BESS $\left(\delta_{i}, \omega_{i}\right)$. These values are directly measured by phasor measurement units (PMUs) installed at generation substations of the system and sent via communication to the regional/super phasor data concentrator (PDC). Hence, it is essential to establish a communication link between PDC and the GI-BESS. For generation plants with more than one generation unit, an equivalent generation unit is assumed, therefore the $i$-index indicates the number of generation plant.

It is essential to note that in this study and for the sake of simplicity, all the communication links are assumed to be time deterministic without any possibility of data collision and delay.

The control diagram which is depicted in Fig. 4 shows the control scheme of a simple POD controller. The main idea behind this controller is taken from [21], which has implemented an POD for SVC. In [21], the generations of the system are divided into two critical and non-critical groups using coherency analysis, then, based on the principles of generalized one-machine infinite bus (GOMIB) system, the $\delta_{\text {GOMIB }}$ and $\omega_{\text {GOMIB }}$ are calculated for each sample of measured $\delta_{i}$ and $\omega_{i}$. These calculations are done by wide area damping controller (WADC) as an application at the control center of WAMS. 


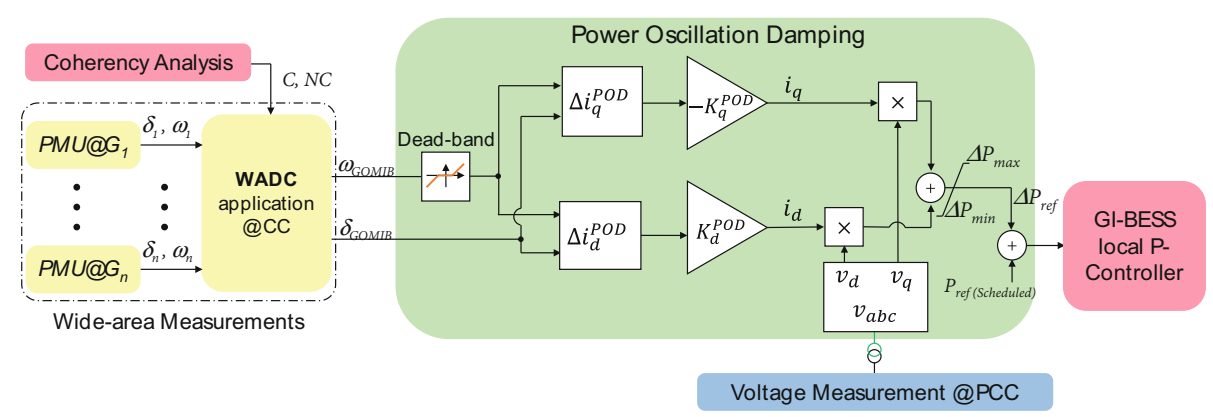

Fig. 4. Proposed wide area controller scheme for POD functionality

The POD block uses CLF as a representative for energy function of the system to mitigate the power oscillation by applying a change in the active power reference of the BESS Converter, $\Delta P_{\text {ref. }}$.

The single machine equivalent (SIME) method from [21] is used to obtain the proposed P-based control laws for the POD function, yielding

$$
\begin{gathered}
i_{q}=-k_{q}^{P O D} \cdot \cos \left(\delta_{G O M I B}\right) \omega_{G O M I B} \\
i_{d}=k_{d}^{P O D} \cdot \sin \left(\delta_{G O M I B}\right) \omega_{G O M I B}
\end{gathered}
$$

where, $i_{q}$ and $i_{d}$ are q- and d-axis currents, $k_{d}^{P O D}$ and $k_{q}^{P O D}$ are time-varying tuneable gains that should be tuned optimally for different operational scenarios, and $\delta_{G O M I B}$ and $\omega_{G O M I B}$ are the angle and angular velocity of GOMIB. The $\omega_{G O M I B}$ and $\delta_{G O M I B}$ are calculated as

$$
\begin{gathered}
\omega_{\text {GOMIB }}=\left(\sum_{i \in C} m_{i}\right)^{-1} \sum_{i \in C} m_{i} \omega_{i}-\left(\sum_{i \in N C} m_{i}\right)^{-1} \Sigma_{j \in N C} m_{j} \omega_{j} \\
\delta_{G O M I B}=\left(\sum_{i \in C} m_{i}\right)^{-1} \Sigma_{i \in C} m_{i} \delta_{i}-\left(\sum_{i \in N C} m_{i}\right)^{-1} \sum_{j \in N C} \delta_{j} \omega_{j}
\end{gathered}
$$

Where subscript $C$ indicates the machines inside the critical group and subscript $N C$ indicates the machines inside non-critical group in the concept of SIME.

Finally, the amount of reactive power to be exchanged by GI-BESS for POD $\left(P_{P O D}=\Delta P_{r e f}\right)$ is calculated using $P_{P O D}=v_{q} \cdot i_{q}+v_{d} \cdot i_{d}$, which is added to the $P_{r e f}$ which is scheduled for the GI-BESS.

\section{Simulation and Results}

To validate the proposed controller, the IEEE 39-bus system is used. The grid is implemented in DIgSILENT Power Factory and it is assumed this network comprises three principle areas, where a $200 \mathrm{MW} / 200 \mathrm{MWh}$ GI-BESS with an initial state of charge (SoC) level of $50 \%$ is installed at Bus 14. The GI-BESS scheduled power is set to inject active power to the grid. 
Regarding the installed capacity of this test system, the capacity of GI-BESS is almost $1 \%$ of total installed capacity (16800 MW) which is an acceptable range for a grid scale BESS.

In order to obtain good POD performance, it is necessary to find the optimal values for $k_{d}^{P O D}$ and $k_{q}^{P O D}$. In this paper, a hybrid optimization based on combination of DE and L-BFGS-B optimization techniques is applied to find the optimal value for $k_{d}^{P O D}$ and $k_{q}^{P O D}$.

As, the main focus of this paper is on the measurement system, the formulation of optimization problem is out of scope and will be discussed in future works. In addition, in this paper and for the sake of simplicity, it is assumed that $k_{d}^{P O D}=k_{q}^{P O D}=k$. The optimal value for $k$ is 381.92 .

It is essential to note that, the optimal value for k changes regarding the system's operation point. Therefore, it is recommended to update the gain value based on the grid status. However, in this paper and for an instance of power service for GI-BESS, just a single operation scenario is discussed.

In this operation scenario, the system undergoes a three-phase short-circuit event on line 14-15 at $\mathrm{t}=1 \mathrm{~s}$, which is cleared after $100 \mathrm{~ms}$, causing an inter-area oscillation on the tie-lines 09-39 and 14-15.

As it depicted in Fig. 5, the FFT analysis of lines flow during the fault indicates the frequency of this oscillation. The modes shapes of this system after modal analysis for $\mathrm{f}=0.645 \mathrm{~Hz}$ shows that two major groups of synchronous generators oscillate against each other.

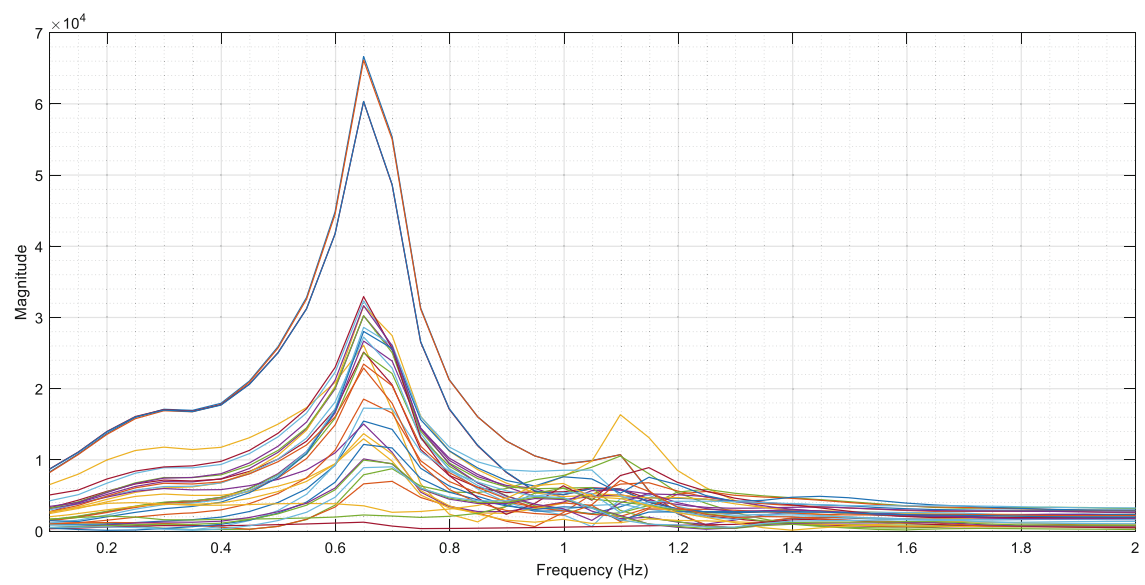

Fig. 5. FFT analysis of active power for all lines of IEEE 39 bus test case system.

Figure 6 depicts the active power of Line 14-15, for three different cases: without GI-BESS, with GI-BESS, and GI-BESS with the proposed controller. However, installing a GI-BESS improves the POD, this figure clearly shows the excellent 


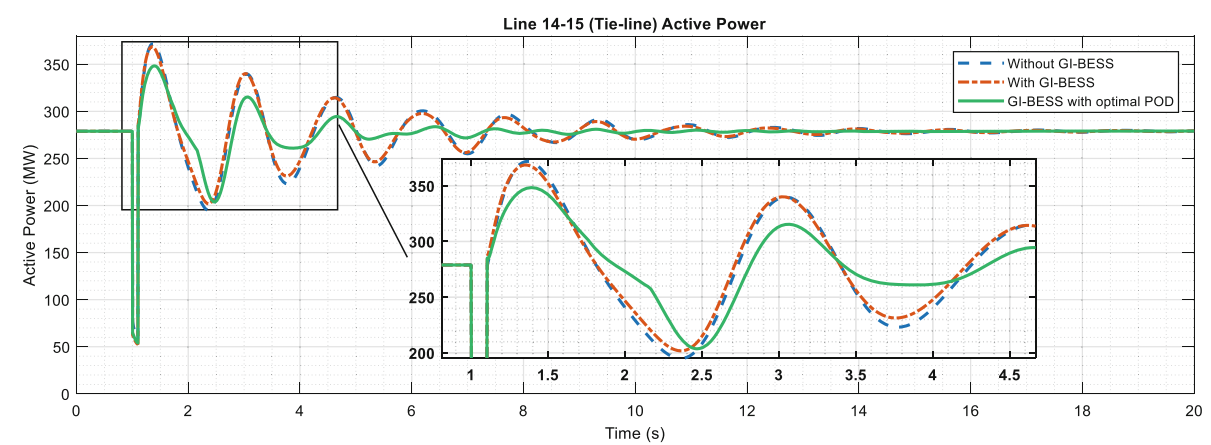

Fig. 6. The active power oscillation of Line 14-15, without GI-BESS and with GI-BESS with and without proposed POD controller.

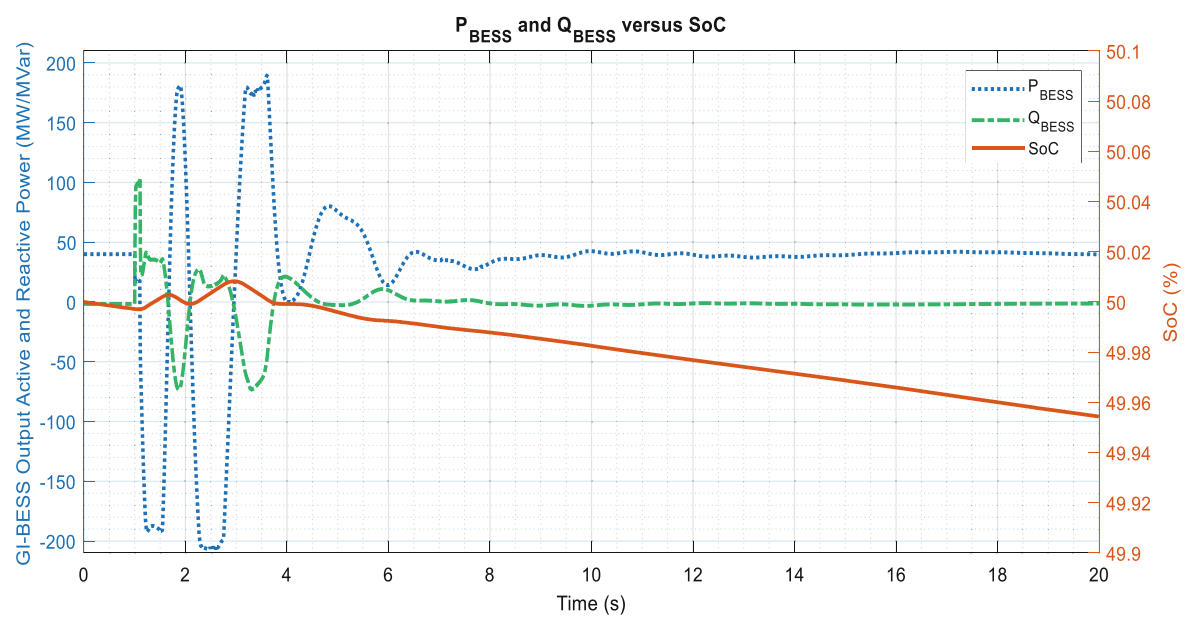

Fig. 7. SoC level versus the GI-BESS active and reactive power exchange.

performance of the proposed controller in regards to shaving the peak and increasing the damping ratio of the system.

For making GI-BESS available to participate in both energy and power services, it is important to assess the amount of stored energy, which could be injected to the grid and the available capacity for absorbing energy from the grid. The SoC can be defined as a good measure to find the effect of power oscillation damping enhancement as a power service on regular energy services of the GI-BESS. Therefore, to assess the effect of the proposed controller on the SoC level of the GI-BESS, a comparison is presented in Fig. 7.

Clearly, the impact of the proposed controller on the SoC level is negligible. This indicated that the GI-BESS was delivering the active power to the grid regarding the dispatch and participating in power oscillation damping simultaneously. 


\section{Conclusion}

This paper presents a practical scheme for integration of SMT into the control of GIESS by delivering real-time situational awareness over the status of the grid. Furthermore, a basic CFL based POD function is implemented as an instance to assess this integration. This integration enables the GI-ESS to participate in both power and energy services of flexibility market, simultaneously. The simulation results prove the efficiency of the proposed monitoring system by showing the negligible impact the participation in power services has on the SoC of the GI-BESS, as well as, the excellent ability of GI-BESS to mitigate low frequency inter-area power oscillations.

\section{References}

1. Atanackovic, D., Clapauch, J.H., Dwernychuk, G., Gurney, J., Lee, H.: First steps to wide area control. IEEE Power Energy Mag. 6(1), 61-68 (2008)

2. Pérez Arriaga, I., Knittel, C., et al: Utility of the future. In: An MIT Energy Initiative Response (2016)

3. Novosel, D., Madani, V., Bhargave, B., Vu, K., Cole, J.: Dawn of the grid synchronization. IEEE Power Energy Mag. 6(1), 49-60 (2008)

4. Bevrani, H., Mitani, Y., Watanabe, M.: Power System Monitoring and Control. Wiley, Hoboken (2014)

5. Lawan, M.M.G., Raharijaona, J., Camara, M.B., Dakyo, B.: Power control for decentralized energy production system based on the renewable energies. In: ICRERA 2017, pp. 11321138 (2017)

6. Tourou, P., Chhor, J., Günther, K., Sourkounis, C.: Energy storage integration in DFIGbased wind energy conversion systems for improved fault ride-through capability. In: ICRERA 2017, pp. 374-377 (2017)

7. Ahn, J.H., Lee, B.K.: A novel parallel control for modular energy storage system achieving high performance, redundancy and applicability. In: ICRERA 2017, pp. 5-8 (2017)

8. Hammad, E., Farraj, A., Kundur, D.: on effective virtual inertia of storage-based distributed control for transient stability. IEEE Trans. Smart Grid 3053(c), 1-10 (2017)

9. Kiaei, I., Lotfifard, S.: Tube-based model predictive control of energy storage systems for enhancing transient stability of power systems. IEEE Trans. Smart Grid 3053(c), 1-10 (2017)

10. Wang, Y., Dvorkin, Y., Fernandez-Blanco, R., Xu, B., Kirschen, D.S.: Impact of local transmission congestion on energy storage arbitrage opportunities. In: IEEE Power Energy Society General Meeting (2018)

11. Kim, M., Kwasinski, A.: Decentralized hierarchical control of active power distribution nodes. IEEE Trans. Energy Convers. 29(4), 934-943 (2014)

12. Torkzadeh, R., Eliassi, M., Mazidi, P., Rodriguez, P.: Synchrophasor measurements for control of grid interactive energy storage system: design alternatives for monitoring system. In: ICRERA 2018, Paris (2018)

13. Aminifar, F., Fotuhi-Firuzabad, M., Safdarian, A., Davoudi, A., Shahidehpour, M.: Synchrophasor measurement technology in power systems: panorama and state-of-the-art. IEEE Access 2, 1607-1628 (2014)

14. Phadke, A.G., Thorp, J.S.: Synchronized Phasor Measurements and their Applications, vol. 1. Springer, Heidelberg (2017) 
15. Ahmadzadeh-Shooshtari, B., Torkzadeh, R., Kordi, M., Marzooghi, H., Eghtedarnia, F.: SG parameters estimation based on synchrophasor data. IET Gener. Transm. Distrib 12, 2958 2967 (2018)

16. Isazadeh, G., Kordi, M., Eghtedarnia, F., Torkzadeh, R.: A new wide area intelligent multiagent islanding detection method for implementation in designed WAMPAC structure. Energy Procedia 141, 443-453 (2017)

17. Hadley, M.D., McBride, J.B., Edgar, T.W., O’Neil, L.R., Johnson, J.D.: Securing wide area measurement systems. US Department Energy (2007)

18. He, M., Vittal, V., Zhang, J.: Online dynamic security assessment with missing PMU measurements: a data mining approach. IEEE Trans. Power Syst. 28(2), 1969-1977 (2013)

19. Torkzadeh, R., Nasr-Azadani, H., Damaki-Aliabad, A.: A genetic algorithm optimized fuzzy logic controller for UPFC in order to damp of low frequency oscillations in power systems. In: ICEE 2014 (2014)

20. Latorre, H.F., Ghandhari, M., Söder, L.: Active and reactive power control of a VSC-HVDC. Electr. Power Syst. Res. 78(10), 1756-1763 (2008)

21. Ghandhari, M.: Application of control Lyapunov functions to static var compensator. In: IEEE International Conference on Control Applications, pp. 1-6 (2002)

Open Access This chapter is licensed under the terms of the Creative Commons Attribution 4.0 International License (http://creativecommons.org/licenses/by/4.0/), which permits use, sharing, adaptation, distribution and reproduction in any medium or format, as long as you give appropriate credit to the original author(s) and the source, provide a link to the Creative Commons license and indicate if changes were made.

The images or other third party material in this chapter are included in the chapter's Creative Commons license, unless indicated otherwise in a credit line to the material. If material is not included in the chapter's Creative Commons license and your intended use is not permitted by statutory regulation or exceeds the permitted use, you will need to obtain permission directly from the copyright holder.

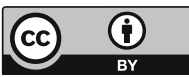

